\title{
Potential Survival Benefit of Anti-Apoptosis Protein: Survivin-Derived Peptide Vaccine with and without Interferon Alpha Therapy for Patients with Advanced or Recurrent Urothelial Cancer-Results from Phase I Clinical Trials
}

\author{
Toshiaki Tanaka, ${ }_{1}^{1}$ Hiroshi Kitamura, ${ }_{1}$ Ryuta Inoue, ${ }^{1}$ Sachiyo Nishida, \\ Akari Takahashi-Takaya, ${ }^{2}$ Sachiyo Kawami, ${ }^{2}$ Toshihiko Torigoe, ${ }^{2}$ Yoshihiko Hirohashi, ${ }^{2}$ \\ Taiji Tsukamoto, ${ }^{1}$ Noriyuki Sato, ${ }^{2}$ and Naoya Masumori ${ }^{1}$ \\ ${ }^{1}$ Department of Urology, Sapporo Medical University School of Medicine, South-1, West-16, Chuo-ku, Sapporo 060-8543, Japan \\ ${ }^{2} 1$ st Department of Pathology, Sapporo Medical University School of Medicine, South-1, West-17, Chuo-ku, Sapporo 060-8556, Japan
}

Correspondence should be addressed to Toshiaki Tanaka; zappa@pop12.odn.ne.jp

Received 5 August 2013; Accepted 30 September 2013

Academic Editor: Pedro A. Reche

Copyright (๑) 2013 Toshiaki Tanaka et al. This is an open access article distributed under the Creative Commons Attribution License, which permits unrestricted use, distribution, and reproduction in any medium, provided the original work is properly cited.

We previously identified a human leukocyte antigen (HLA)-A24-restricted antigenic peptide, survivin-2B80-88, a member of the inhibitor of apoptosis protein family, recognized by CD8+cytotoxic T lymphocytes (CTL). In a phase I clinical trial of survivin2B80-88 vaccination for metastatic urothelial cancer (MUC), we achieved clinical and immunological responses with safety. Moreover, our previous study indicated that interferon alpha (IFN $\alpha$ ) enhanced the effects of the vaccine for colorectal cancer. Therefore, we started a new phase I clinical trial of survivin-2B80-88 vaccination with IFN $\alpha$ for MUC patients. Twenty-one patients were enrolled and no severe adverse event was observed. HLA-A24/survivin-2B80-88 tetramer analysis and ELISPOT assay revealed a significant increase in the frequency of the peptide-specific CTLs after vaccination in nine patients. Six patients had stable disease. The effects of IFN $\alpha$ on the vaccination were unclear for MUC. Throughout two trials, 30 MUO patients received survivin-2B80-88 vaccination. Patients receiving the vaccination had significantly better overall survival than a comparable control group of MUO patients without vaccination $(P=0.0009)$. Survivin-2B80-88 vaccination may be a promising therapy for selected patients with MUC refractory to standard chemotherapy. This trial was registered with UMIN00005859.

\section{Introduction}

Urothelial carcinoma of the bladder is the fourth most common cancer in men [1]. Systemic chemotherapy has been the mainstay of management for metastatic urothelial cancer $[2,3]$, and cisplatin-based combinations have evolved as the standard first-line therapy. The regimens consisting of methotrexate, vinblastine, doxorubicin, and cisplatin (MVAC) and gemcitabine and cisplatin (GC) are currently employed and provide prolongation of survival up to 14.8 and 13.8 months, respectively [3]. However, no standard therapy has been established for patients with progressive disease after the first-line chemotherapy $[2,3]$, and some new regimens including other anticancerous agents such as paclitaxel, ifosphamide, nedaplatin, and vinflunine are used in this setting [4-6], although they have not been proven to have sufficient clinical efficacy.

On the other hand, during the past two decades, research on human tumor immunology and cancer immunotherapy has progressed. Immunization with peptides derived from cancer-specific antigen induces antitumor cytotoxic T lymphocytes (CTLs) [7-9]. A large number of cancer-specific antigens have been identified from melanomas and other cancers, and clinical trials of peptide-based immunotherapy have been carried out.

We previously reported that survivin and its splicing variant survivin-2B were expressed abundantly in various cancer tissues and cancer cell lines, including urothelial cancer, 


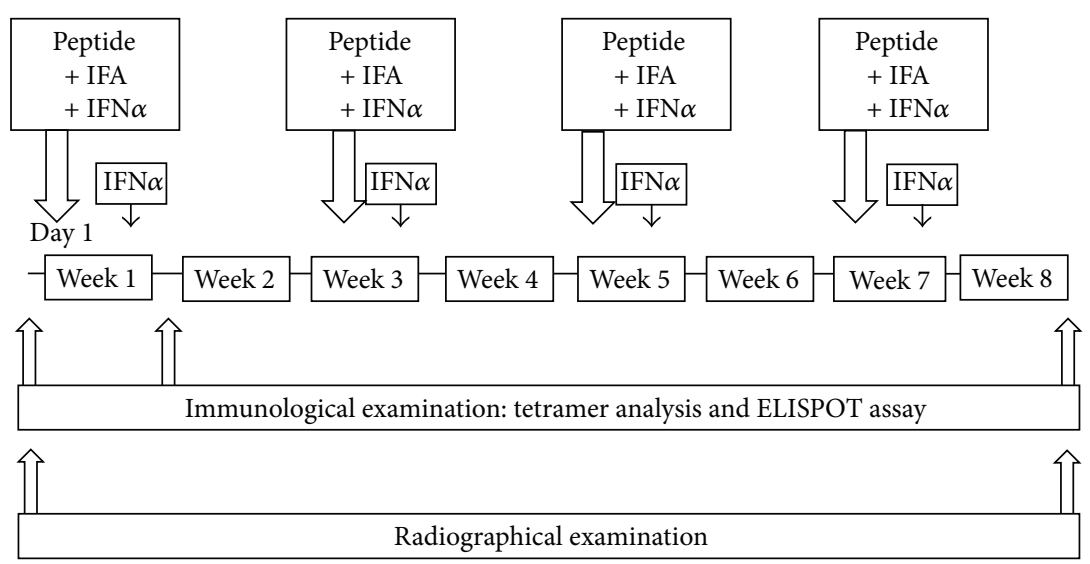

FIGURE 1: Protocol of Study 2. The protocol consisted of survivin-2B80-88 peptide, IFA, and IFN $\alpha$. IFA: incomplete Freund's adjuvant; IFN: interferon.

and were suitable as target antigens for active-specific anticancer immunization [10]. Subsequently, we identified the human leukocyte antigen (HLA)-A24-restricted antigenic peptide survivin-2B80-88 (AYACNTSTL) derived from the exon $2 \mathrm{~B}$-encoded region and recognized by CTLs in the context of HLA-A24 molecules. In addition, we reported further evidence that the survivin-2B80-88 peptide might serve as a potent immunogenic cancer vaccine for various cancers, including bladder cancer [11]. On the basis of these studies, we started a phase I clinical study using survivin2B80-88 peptide vaccination for urothelial cancers (Study 1) [12]. This study revealed that survivin-2B80-88 peptide vaccination was safe and well tolerated without severe side effects and could induce survivin-2B80-88 peptide-specific CTLs. Moreover, we previously reported that combination with interferon (IFN) alpha successfully enhanced the immunological responses of patients who received survivin2B80-88 peptide vaccination for colorectal [13] and pancreatic cancers [14]. Therefore we conducted a phase I clinical study of survivin-2B80-88 peptide vaccination in combination with IFN alpha for patients with advanced or recurrent urothelial cancer expressing survivin to assess the safety and immunological efficacy (Study 2). In addition, we analyzed the effects on survival of survivin-2B80-88 peptide vaccination therapy with and without IFN alpha using the pooled data of Study 1 and Study 2.

\section{Materials and Methods}

2.1. Patient Selection. The study protocol was approved by the Clinical Institutional Ethical Review Board of the Medical Institute of Bioregulation, Sapporo Medical University, Japan. The HLA-A typing and immunohistochemical study were performed after obtaining informed consent from all candidate patients. Patients enrolled in this study were required to conform to the following criteria: (1) histologically proven urothelial cancer, (2) HLA-A $* 2402$ positive, (3) survivinand HLA class I-positive carcinomatous lesions on the primary site demonstrated by immunohistochemistry, (4) age between 20 and 85 years old, (5) surgical excision of the primary tumor, and (6) Eastern Cooperative Oncology Group
(ECOG) performance status between 0 and 3. Exclusion criteria included (1) prior cancer therapy such as chemotherapy, radiation therapy, steroid therapy, or other immunotherapies within the previous 4 weeks, (2) the presence of other cancers that might influence the prognosis, (3) immunodeficiency or a history of splenectomy, (4) severe cardiac insufficiency, acute infection, or hematopoietic failure, and (5) unsuitability for the trial based on clinical judgment. This study was carried out at the Department of Urology, Sapporo Medical University Hospital from May 2009 to June 2013.

2.2. Peptide Preparation. The peptide, survivin-2B80-88 with the sequence AYACNTSTL, was prepared under good manufacturing practice conditions by Multiple Peptide Systems (San Diego, CA, USA) [12-14]. The identity of the peptide was confirmed by mass spectrometry analysis and the purity was shown to be more than $98 \%$ as assessed by high-pressure liquid chromatography analysis. The peptide was supplied as a freeze-dried, sterile white powder. It was dissolved in $1.0 \mathrm{~mL}$ of physiological saline (Otsuka Pharmaceutical Co., Ltd, Tokyo, Japan) and stored at $-80^{\circ} \mathrm{C}$ until just before use.

2.3. IFA and IFN Alpha Preparation. Montanide ISA 51 (Seppic, Paris, France) was used as IFA. Human IFN alpha was purchased from Dainippon-Sumitomo Pharmaceutical Co. (Osaka, Japan).

2.4. Patient Treatment. In Study 1 we administered the survivin-2B80-88 peptide plus IFA [12]. In Study 2, the survivin-2B80-88 peptide plus IFA and a type-I IFN, IFN alpha, were used as illustrated in Figure 1. The doses were determined according to previous studies $[13,14]$. Survivin2B80-88 at a dose of $1 \mathrm{mg} / 1 \mathrm{~mL}$ and IFA at a dose of $1 \mathrm{~mL}$ were mixed immediately before vaccination. The patients were then vaccinated subcutaneously four times at 14-day intervals. In addition, IFN alpha at a dose of 3,000,000 IU was administered subcutaneously immediately before vaccination and three days after vaccination at the site of vaccination. The primary endpoint was safety. The secondary endpoints were investigations about antitumor effects and clinical and immunological monitoring. 
TABle 1: Profiles of patients with advanced urothelial cancer enrolled in Study 2.

\begin{tabular}{|c|c|c|c|c|c|c|}
\hline No. & Age & Sex & Primary site & Recurrence site & ECOG PS & Prior chemotherapy (number of cycles) \\
\hline 1 & 72 & $\mathrm{f}$ & UUT & LN in neck, mediastinum, and abdomen & 1 & MVAC (5), TIN (2) \\
\hline 2 & 36 & $\mathrm{~m}$ & Bladder & Abdominal LN Bone & 0 & MVAC (2), TIN (1) \\
\hline 3 & 61 & $\mathrm{~m}$ & UUT & Pelvic soft tissue & 0 & GC (3), TIN (2) \\
\hline 4 & 75 & $\mathrm{~m}$ & Bladder & Abdominal LN & 0 & MVAC (2) \\
\hline 5 & 76 & $\mathrm{~m}$ & UUT & Renal pelvis, urethra & 2 & GC (3), TIN (1) \\
\hline 6 & 60 & $\mathrm{f}$ & UUT & Abdominal LN & 1 & MVAC (3) \\
\hline 7 & 72 & $\mathrm{f}$ & UUT & Mediastinal LN & 1 & $\operatorname{MEC}(1), \operatorname{GEM}(1), \mathrm{TIN}(2)$ \\
\hline 8 & 77 & $\mathrm{~m}$ & Bladder & Lung & 0 & None \\
\hline 9 & 75 & $\mathrm{f}$ & UUT & Pelvic soft tissue & 1 & None \\
\hline 10 & 68 & $\mathrm{~m}$ & UUT & Abdominal LN Lung & 0 & GC (4) \\
\hline 11 & 72 & $\mathrm{~m}$ & Bladder & Pelvic LN, liver & 0 & TG (1) \\
\hline 12 & 58 & $\mathrm{~m}$ & Bladder & LN in neck, abdomen, and pelvis & 0 & GC (2), TIN (2) \\
\hline 13 & 64 & $\mathrm{~m}$ & Bladder & LN in abdomen and pelvis & 1 & GC (2) \\
\hline 14 & 73 & $\mathrm{f}$ & Bladder & Lung, liver, and bone & 0 & GC (3), TIN (2) \\
\hline 15 & 62 & $\mathrm{~m}$ & Bladder & Lung & 1 & GC (1), GCar (1) \\
\hline 16 & 74 & $\mathrm{~m}$ & Bladder & Abdominal LN & 2 & GC (4), TIN (2) \\
\hline 17 & 53 & $\mathrm{~m}$ & UUT & Lung, subcutaneous & 2 & None \\
\hline 18 & 61 & $\mathrm{~m}$ & Bladder & Lung & 1 & GC (6), TIN (3) \\
\hline 19 & 56 & $\mathrm{~m}$ & Bladder & Abdominal LN, liver & 1 & GCar (2) \\
\hline 20 & 63 & $\mathrm{f}$ & Bladder & Abdominal LN, lung, and liver & 1 & GC (4) \\
\hline 21 & 73 & $\mathrm{~m}$ & Bladder & Abdominal LN, liver & 0 & GC (4) \\
\hline
\end{tabular}

UUT: upper urinary tract; LN: lymph node; ECOG PS: Eastern Cooperative Oncology Group performance status; MVAC: methotrexate, vinblastine, adriamycin, and cisplatin; TIN: paclitaxel, ifosphamide, and nedaplatin; GC: gemcitabine and cisplatin; MEC: methotrexate, etoposide, and cisplatin; GEM: gemcitabine; TG: paclitaxel and gemcitabine; GCar: gemcitabine and carboplatin.

2.5. Toxicity Evaluation. Patients were examined closely for signs of toxicity during and after vaccination. Adverse events were recorded using the National Cancer Institute Common Terminology Criteria for Adverse Events version 4.0 (CTCAE v4.0) [15].

2.6. Clinical Response Evaluation. Physical examinations and hematological examinations were conducted before and after each vaccination [12-14]. Immunohistochemical study of the HLA class I expression in patients' primary urothelial cancer tissues was done with anti-HLA class I heavy chain monoclonal antibody EMR-8-5 (Funakoshi Co., Tokyo, Japan). We evaluated tumor size using CT scans or MRI by comparing the size before the first vaccination with that after the fourth vaccination. A complete response (CR) was defined as complete disappearance of all measurable and evaluable diseases. A partial response (PR) was defined as a $\geq 30 \%$ decrease from baseline in the size of all measurable lesions (sum of maximal diameters). Progressive disease (PD) was defined as an increase in the sum of maximal diameters by at least $20 \%$ or the appearance of new lesions. Stable disease (SD) was defined as the absence of criteria matching those for CR, PR, or PD [12-14].

2.7. In Vitro Stimulation of PBMC. PBMCs were isolated from blood samples by Ficoll-Conray density gradient centrifugation. They were then frozen and stored at $-80^{\circ} \mathrm{C}$. As needed, frozen PBMCs were thawed and incubated in the presence of $30 \mu \mathrm{g} / \mathrm{mL}$ survivin-2B80-88 in AIM-V medium containing
$10 \%$ human serum at room temperature. Next, interleukin-2 was added at a final concentration of $50 \mathrm{U} / \mathrm{mL} 1 \mathrm{~h}, 2$ days, 4 days, and 6 days after the addition of the peptide. On day 7 of culture, the PBMCs were analyzed by tetramer staining and ELISPOT assay.

2.8. Tetramer Staining. FITC-labeled HLA-A*2402-human immunodeficiency virus (HIV) peptide (RYLRDQQLL) and PE-labeled HLA-A*2402-survivin-2B80-88 peptide tetramers were purchased from MBL, Inc. (Nagoya, Japan). For flow cytometric analysis, PBMCs, which were stimulated in vitro as above, were stained with the PE-labeled tetramer at $37^{\circ} \mathrm{C}$ for $20 \mathrm{~min}$, followed by staining with an FITCconjugated anti-CD $8 \mathrm{mAb}$ (Beckton Dickinson Biosciences, San Jose, CA, USA) at $4^{\circ} \mathrm{C}$ for $30 \mathrm{~min}$. Cells were washed twice with $\mathrm{PBS}$ before fixation in $1 \%$ formaldehyde. Flow cytometric analysis was performed using FACSCalibur and CellQuest software (Beckton Dickinson Biosciences, San Jose, CA, USA). The frequency of CTL precursors was calculated as the number of tetramer-positive cells divided by the number of CD8-positive cells [12-14].

2.9. ELISPOT Assay. ELISPOT plates were coated sterilely overnight with an IFN-c capture antibody (Beckton Dickinson Biosciences) at $4^{\circ} \mathrm{C}$. The plates were then washed once and blocked with AIM-V medium containing 10\% human serum for $2 \mathrm{~h}$ at room temperature. CD8-positive $\mathrm{T}$ cells separated from patients' PBMC $\left(5 \times 10^{3}\right.$ cells/well $)$, which were stimulated in vitro as above, were then added to each 
TABLE 2: Summary of clinical and immunological responses to vaccination with survivin-2B80-88 peptide, IFA, and IFN alpha.

\begin{tabular}{|c|c|c|c|c|c|c|}
\hline \multicolumn{2}{|c|}{ No. Adverse events (Grade) ${ }^{*}$} & \multirow{2}{*}{$\begin{array}{c}\text { Tetramer staining }^{\dagger} \\
\text { After/before vaccination } \\
3100 / 3300\end{array}$} & \multirow{2}{*}{$\begin{array}{c}\text { ELISPOT }^{\ddagger} \\
\text { After/before vaccination } \\
98 / 3\end{array}$} & \multirow{2}{*}{$\begin{array}{c}\text { Clinical response } \\
\mathrm{PD}\end{array}$} & \multirow{2}{*}{$\begin{array}{c}\text { Followup (months) } \\
6.5\end{array}$} & \multirow{2}{*}{$\begin{array}{c}\text { Outcome } \\
\text { DOD }\end{array}$} \\
\hline 1 & Fever (1) & & & & & \\
\hline 2 & Fever (1) & $2700 / 600$ & $31 / 20$ & $\mathrm{SD}$ & 14.5 & DOD \\
\hline 3 & Fever (1) & $4400 / 600$ & $62 / 36$ & $\mathrm{SD}$ & 17.0 & DOD \\
\hline 4 & $\begin{array}{l}\text { Fever (1) } \\
\text { Induration at injection site (1) }\end{array}$ & $16400 / 1700$ & $49 / 12$ & SD & 32.5 & AWD \\
\hline 5 & Fever (1) & $500 / 10900$ & $29 / 8$ & $\mathrm{PD}$ & 2.0 & DOD \\
\hline 6 & Fever (1) & $2000 / 300$ & $32 / 29$ & $\mathrm{PD}$ & 4.0 & DOD \\
\hline 7 & Induration at injection site (1) & $4100 / 0$ & $41 / 15$ & $\mathrm{PD}$ & 6.5 & DOD \\
\hline 8 & Fever $(1)$ & $2100 / 2000$ & $49 / 33$ & $\mathrm{PD}$ & 14.5 & DOD \\
\hline 9 & Fever (1) & $2000 / 500$ & $65 / 21$ & SD & 7.0 & DOD \\
\hline 10 & Fever (1) & $0 / 4500$ & $53 / 6$ & $\mathrm{PD}$ & 6.5 & AWD \\
\hline 11 & None & $38900 / 0$ & $10 / 0$ & PD & 10.0 & DOD \\
\hline 12 & Fever (1) & $2400 / 0$ & $117 / 80$ & SD & 6.0 & AWD \\
\hline 13 & Fever (1) & $0 / 0$ & $28 / 9$ & PD & 9.5 & DOD \\
\hline 14 & None & $1200 / 800$ & $95 / 14$ & $\mathrm{PD}$ & 4.0 & AWD \\
\hline 15 & Fever (1) & $1600 / 200$ & $616 / 68$ & SD & 8.5 & AWD \\
\hline 16 & None & $700 / 300$ & $11 / 63$ & PD & 5.5 & DOD \\
\hline 17 & Fever (1) & $200 / 400$ & $39 / 0$ & $\mathrm{PD}$ & 5.5 & AWD \\
\hline 18 & $\begin{array}{c}\text { Fever (1) } \\
\text { Induration at injection site (1) }\end{array}$ & $3700 / 4600$ & $61 / 32$ & $\mathrm{PD}$ & 4.0 & AWD \\
\hline 19 & None & $1400 / 200$ & $7 / 5$ & $\mathrm{PD}$ & 1.0 & AWD \\
\hline 20 & None & $900 / 800$ & $25 / 0$ & PD & 2.0 & AWD \\
\hline 21 & None & $2000 / 300$ & $0 / 0$ & $\mathrm{PD}$ & 2.0 & AWD \\
\hline
\end{tabular}

${ }^{*}$ Adverse events were recorded using the National Cancer Institute Common Terminology Criteria for Adverse Events version 4.0 (CTCAE v4.0). ${ }^{\dagger}$ Cytotoxic T-lymphocyte (CTL) frequency before and after treatment in patients was assessed with an HLA-A24-restricted survivin-2B80-88 (AYACNTSTL) peptide tetramer. An HLA-A24-restricted HIV peptide (RYLRDQQLL) tetramer was used as a negative control. The number of survivin-2B80-88 peptide tetramerpositive but HIV peptide-negative CTLs in $10^{4} \mathrm{CD} 8 \mathrm{~T}$ cells is shown. ${ }^{\ddagger}$ Interferon gamma secretion of pre- and postvaccinated patients' CD8 T cells was assessed with enzyme-linked immunosorbent spot (ELISPOT) assay using T2-A24 cells pulsed with survivin-2B80-88 peptide. The numbers of spots in $5 \times 10^{3} \mathrm{CD} 8 \mathrm{~T}$ cells are shown. SD: stable disease; PD: progressive disease; DOD: dead of disease; AWD: alive with disease.

well along with HLA-A24-transfected CIR cells (CIR-A24) $\left(5 \times 10^{4}\right.$ cells/well), which had been preincubated with or without survivin-2B80-88 $(10 \mathrm{mg} / \mathrm{mL})$ or with an HIV peptide as a negative control. After incubation in a $5 \%$ $\mathrm{CO}_{2}$ humidified chamber at $37^{\circ} \mathrm{C}$ for $24 \mathrm{~h}$, the wells were washed vigorously five times with PBS and incubated with a biotinylated anti-human IFN-c antibody and horseradish peroxidase-conjugated avidin. Spots were visualized and analyzed using KS ELISPOT (Carl Zeiss, Jena, Germany). In the present study, the cutoff point for ELISPOT was determined according to previous studies; positive ( + ) ELISPOT represented a more than twofold increase of survivin-2B8088 peptide-specific CD8 T cell IFNc-positive spots compared with HIV peptide-specific CD8 T-cell spots, whereas negative $(-)$ represented a less than twofold increase $[13,14]$.

2.10. Statistical Analysis. Continuous variables were compared using the Student's $t$-test. Given the small size, we confirmed all results with the Mann-Whitney $U$ test. Categorized variables were compared using Fisher's exact probability test. Overall survival rates (OS) were evaluated by the KaplanMeier method, and differences between two groups were compared using the log-rank test and Cox proportional hazards regression models. A value of $P<0.05$ was considered to indicate statistical significance. The calculations were performed using Statview 5.0 (SAS Institute, Cary, NC).

\section{Results}

3.1. Patient Profile. Twenty-one patients were enrolled in Study 2 (Table 1). They consisted of 15 men and 6 women, whose age range was 36-77 years. Three patients did not receive chemotherapy before vaccination because they were unfit for cisplatin-based chemotherapy due to impaired renal function.

3.2. Safety. Six patients (cases 5, 6, 16, 17, 19, and 20) discontinued halfway through the protocol because of disease progression. The remaining 15 patients received the complete regimen including four vaccinations. None of the treatment interruptions was due to adverse effects of the vaccination. Peptide vaccination was well tolerated in all 21 patients. As shown in Table 2, no hematologic, cardiovascular, hepatic, or renal toxicity was observed. No other severe adverse events 


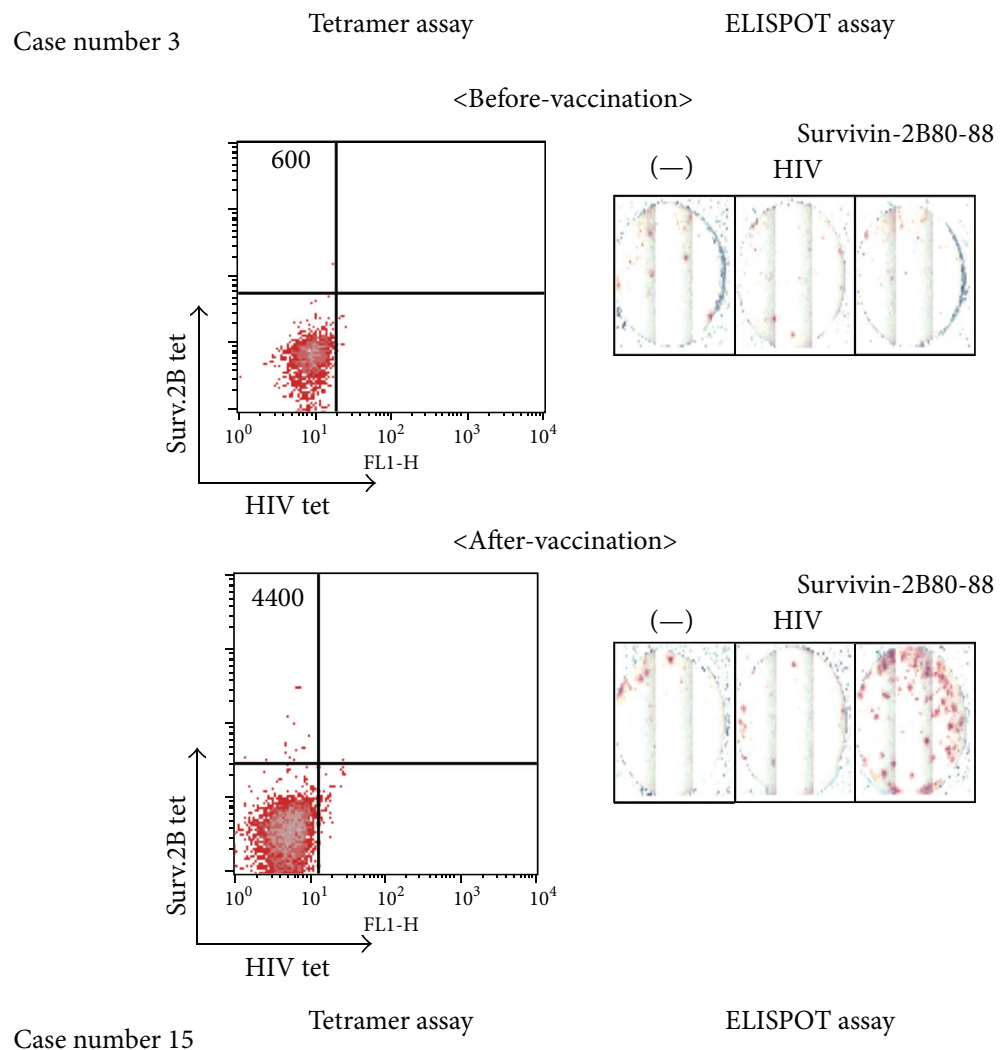

Case number 15
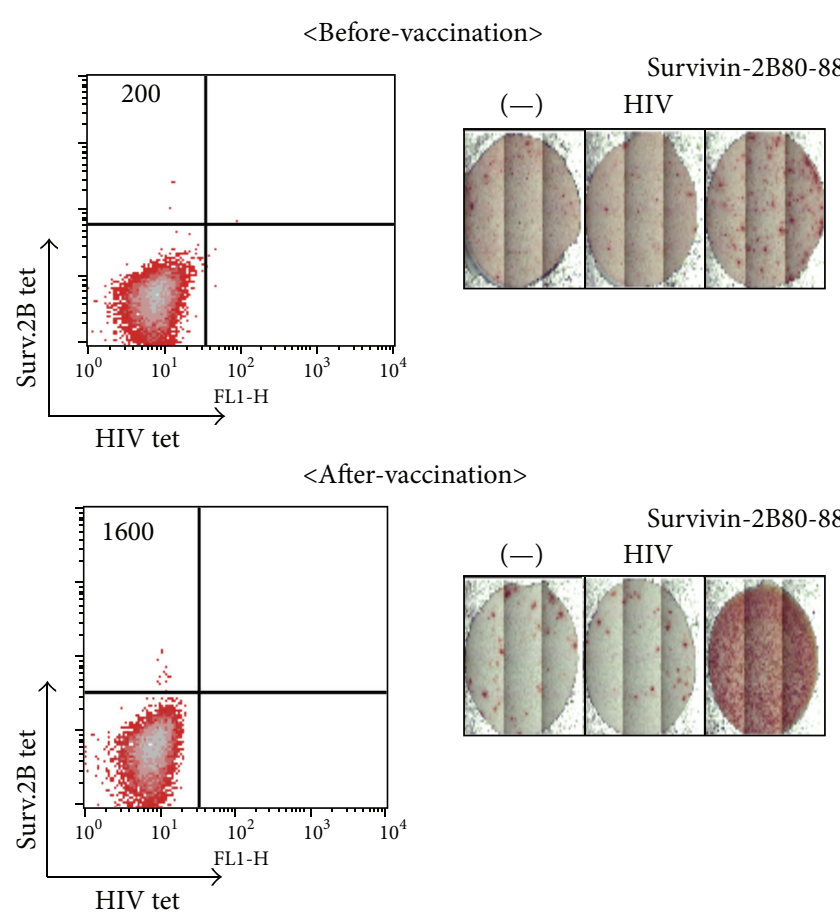

FIGURE 2: Representative illustration of immunological analysis in patients 3 and 15 who were treated with survivin-2B80-88 plus IFA with IFN alpha. Tetramer and ELISPOT analyses before and after vaccinations. The number in the tetramer analysis indicates survivin-2B80-88 peptide-specific CD8+ T cells among $10^{4} \mathrm{CD} 8+\mathrm{T}$ cells. ELISPOT: enzyme-linked immunosorbent spot; HIV: human immunodeficiency virus; HLA: human leukocyte antigen; IFA: incomplete Freund's adjuvant; IFN: interferon. 
TABLe 3: Baseline characteristics and immunological responses in Study 1 and Study 2.

\begin{tabular}{lccr}
\hline & Study 1 & Study 2 & $P$ value \\
\hline$n$ & 9 & 21 & $05.7 \pm 10.3$ \\
Age & $58.1 \pm 9.1$ & $15 / 6$ & 0.0593 \\
Sex (male/female) & $4 / 5$ & $11 / 10$ & 0.2252 \\
Primary site (UUT/bladder) & $1 / 8$ & $11(52.4 \%)$ & 0.0492 \\
Visceral metastases & $4(44.4 \%)$ & $18(85.7 \%)$ & 0.9999 \\
Prior chemotherapy & $9(100 \%)$ & $10 / 9 / 2$ & 0.2320 \\
ECOG PS (0/1/2) & $4 / 5 / 0$ & $9(42.8 \%)$ & 0.9999 \\
Induction of CTLs & $5(71.4 \%)^{*}$ & $6(28.6 \%)$ & 0.3845 \\
Non-PD in clinical response & $2(33.3 \%)^{\dagger}$ & 0.6424 \\
\hline
\end{tabular}

${ }^{*}$ Immunological results were obtained in 7 of 9 cases. ${ }^{\dagger}$ Clinical responses were assessed in 6 cases. UUT: upper urinary tract; ECOG PS: Eastern Cooperative Oncology Group performance status; CTL: cytotoxic T-lymphocyte; PD: progressive disease.

were observed during or after vaccination. As minor side effects, 14 patients (cases 1-6, 8-10,12,13,15, 17, and 18) developed grade 1 fever, possibly due to IFN alpha, and 3 patients (cases 4, 7, and 18) developed grade 1 local skin reactions with redness and induration at the injection sites. No other severe adverse events were observed during or after vaccination.

3.3. Immunological and Clinical Responses. Representative illustrations of immunological analysis in cases 3 and 15 are shown in Figure 2, and Table 2 summarizes the immunological and clinical results. HLA-A24/survivin-2B80-88 peptide tetramer analysis revealed a significant increase in the peptide-specific CTL frequency of CD8-positive T cells after vaccination in 13 patients (cases $2,3,4,6,7,9,11,12,14,15,16$, 19, and 21), as shown in Table 2. Of them, however, cases 6, 16, 19 , and 21 were negative in the ELISPOT study. Thus, functional peptide-specific CTLs were induced in nine patients $(42.8 \%)$ by this vaccination protocol. Radiographical examination revealed SD after four vaccinations in six patients (28.6\%). All of them had an increase in the peptide-specific CTLs proven in both tetramer analysis and ELISPOT assay.

3.4. Impact of IFN Alpha in Combination with the Survivin2B80-88 Peptide on Immunological Responses and Survival. To assess the effect of additional IFN alpha, immunological and clinical outcomes were compared between Study 1 and Study 2. Baseline characteristics and immunological and clinical responses are shown in Table 3. There were no significant differences in either the induction of peptidespecific CTLs or radiographical responses. Furthermore, OS showed no significant difference between the two groups (Figure 3).

3.5. Impact of the Survivin-2B80-88 Peptide Vaccination with and without IFN Alpha on Survival. A total of 30 patients underwent the survivin-2B80-88 peptide vaccination in Study 1 and Study 2. During the course of these studies, 14 patients were excluded due to an ineligible HLA type and 4 patients eventually decided not to receive vaccination although eligible. These 18 patients were evaluated as a control group. Clinical characteristics were comparable between the vaccination group and control group, as shown in Table 4.

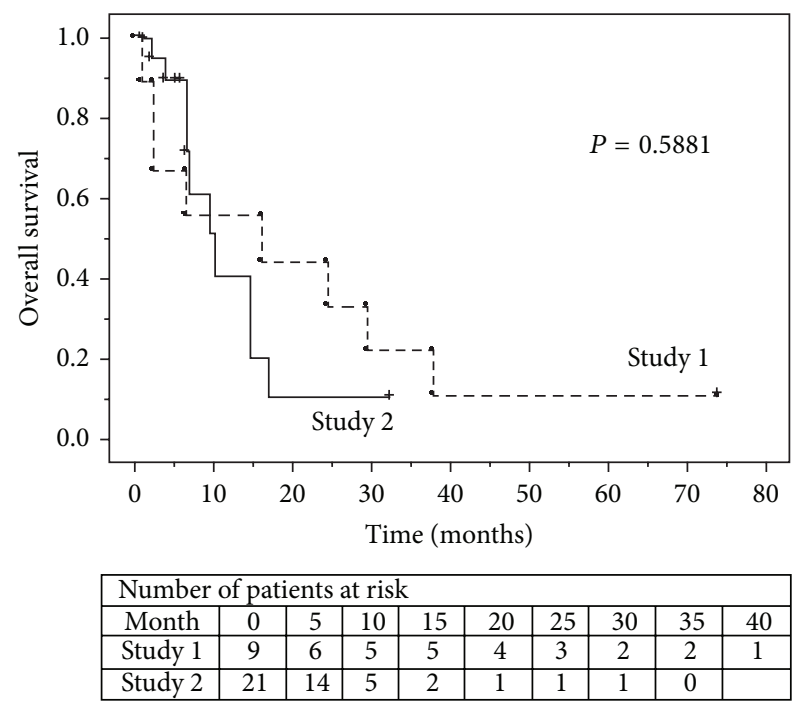

FIGURE 3: Kaplan-Meier estimated overall survival is shown for patients treated with survivin-2B80-88 peptide plus IFA (Study 1) versus survivin-2B80-88 peptide plus IFA in combination with IFA alpha (Study 2). IFA: incomplete Freund's adjuvant; IFN: interferon.

The vaccination group had significantly better OS than the control group $(P=0.0009)$, as shown in Figure 4. Median survival times were 10.0 months and 4.5 months in the vaccination group and control group, respectively. Table 5 lists the results of proportional hazards regression analysis used to test the predictive value of each variable for OS. In this multivariate model adjusted for age, ECOG PS, and the presence of visceral metastases, vaccination therapy was an independent predictive factor for better OS $(P=0.0088)$.

\section{Discussion}

Survivin-2B80-88 vaccination therapy is safe and confers induction of peptide-specific CTLs in patients with metastatic urothelial cancers according to the results of Study 1 [12]. In Study 2, we used a combination protocol of survivin peptide vaccination with IFN alpha in an attempt to enhance the immunogenicity, as with colorectal [13] and pancreatic cancers [14]. The protocol was safe and well tolerated with no 
TABLE 4: Clinical characteristics of vaccination group and control group.

\begin{tabular}{lccc}
\hline & Vaccination group & Control group & $P$ value \\
\hline$n$ & 30 & $66.4 \pm 10.3$ & 0.3355 \\
Age & $63.5 \pm 10.2$ & $15 / 3$ & 0.1956 \\
Sex (male/female) & $19 / 11$ & $12(66.7 \%)$ & 0.3693 \\
Visceral metastases & $15(50.0 \%)$ & $1.00 \pm 0.84$ & 0.1417 \\
Number of visceral metastatic sites & $0.63 \pm 0.76$ & $17(94.4 \%)$ & 0.9999 \\
Prior chemotherapy & $27(90.0 \%)$ & & \\
ECOG PS & & $8(44.4 \%)$ & 0.4002 \\
$\quad 0$ & $13(43.3 \%)$ & $6(33.3 \%)$ & $4(22.3 \%)$ \\
\hline
\end{tabular}

ECOG PS: Eastern Cooperative Oncology Group performance status.

TABLE 5: Multivariate proportional hazards regression model for overall survival.

\begin{tabular}{lccc}
\hline Variable & HR & $95 \%$ CI & $P$ value \\
\hline Age: $<65$ versus $\geq 65$ years & 0.782 & $0.343-1.784$ & 0.5591 \\
ECOG PS: 0 versus $\geq 1$ & 0.335 & $0.160-0.703$ & 0.0038 \\
Visceral metastases: no versus yes & 0.599 & $0.284-1.263$ & 0.1782 \\
Vaccination: yes versus no & 0.308 & $0.127-0.743$ & 0.0088 \\
\hline
\end{tabular}

ECOG PS: Eastern Cooperative Oncology Group performance status; HR: hazard ratio; CI: confidence interval.

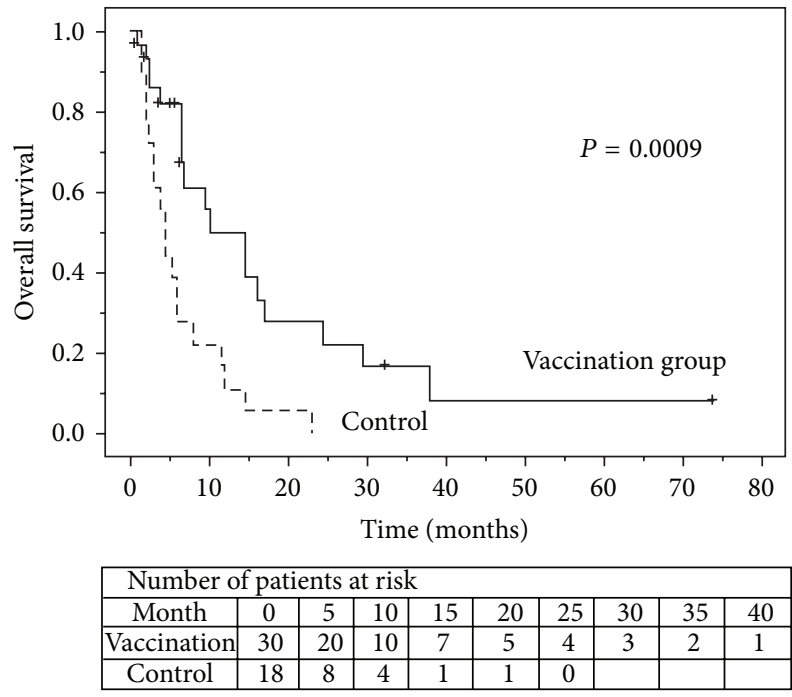

Figure 4: Kaplan-Meier estimated overall survival (OS) is shown for patients who received survivin-2B80-88 peptide vaccination with and without IFN alpha and did not receive survivin-2B80-88 peptide vaccination. A statistically significant difference in OS was identified between the two groups.

severe adverse effects, as in the case of colorectal and pancreatic cancers $[13,14]$. Fever was the most frequent adverse effect and may have resulted from the use of interferon alpha. However, we could not find any benefit of additional interferon alpha in urothelial cancer patients in terms of enhancing the immunogenicity of the survivin-2B80-88 peptide. In a previous study, peptide-specific CTLs were induced in $50 \%$ of patients with colorectal cancer by survivin-2B8088 peptide vaccination in combination with interferon alpha, but in $0 \%$ by that without interferon alpha [13]. On the other hand, survivin peptide vaccination without interferon alpha induced peptide-specific CTLs in $67 \%$ of urothelial cancer patients [12]. IFN alpha may be an insufficient adjuvant for further enhancement of the immunogenicity of survivin$2 \mathrm{~B} 80-88$, and improvement of the protocol is required. Besides IFN alpha, other cytokines such as interleukin (IL)-2, IL-4, IL-15, and granulocyte macrophage colony-stimulating factor are expected to have effects leading to stronger immune responses in both the induction and effector phases [16]. Furthermore, blockade of negative regulation of the immune response is considered to be an important strategy to enhance the immunological and clinical responses of peptide vaccination therapy $[16,17]$. Low-dose cyclophosphamide $[18,19]$ and an anti-CD25 [20] antibody are employed to suppress regulatory $\mathrm{T}$ cells, and low-dose gemcitabine is promising to suppress myeloid-derived suppressor cells [21]. These agents might be effective to enhance the immunological response and clinical efficacy in survivin-2B-80-88 peptide vaccination therapy for urothelial cancer.

In cancer vaccination therapy, tumor shrinkage is not expected and may not be an appropriate endpoint for evaluation of the efficacy of cancer immunotherapy [22]. Although neither CR nor PR after the vaccination was observed in our series, all six patients with SD also had increases in CTLs. SD can be considered to be a result of immunological responses to the survivin-2B80-88 peptide vaccine. Therefore, the results of the current study suggest that survivin2B80-88 peptide vaccination therapy potentially provides survival benefit for patients with metastatic urothelial cancer. However, this study had only a small number of subjects. Although the control group was comparable, patients were not randomized. To confirm the efficacy for survival, a larger randomized clinical trial is necessary. 
There is no standard therapy for metastatic urothelial cancers refractory to standard chemotherapy [2, 3]. In addition, most second-line chemotherapy regimens under investigation have severe adverse events, which can impair patients' quality of life and are often associated with lifethreatening adverse effects [4-6]. On the other hand, the results of the current study suggest that survivin-2B80-88 peptide vaccination therapy is safe and well tolerated and may potentially have clinical benefits in selected patients. Thus, survivin-2B80-88 peptide vaccination appears to be a promising treatment strategy for metastatic urothelial cancers refractory to standard chemotherapy.

\section{Conclusions}

Although survivin-2B80-88 peptide vaccination in combination with IFN alpha is safe and well tolerated, the effects of additional IFN alpha are unclear. According to the results of pooled data analysis of Study 1 and Study 2, survivin-2B8088 peptide vaccination therapy potentially has clinical effects; thus, it may be a promising therapy for selected patients with metastatic urothelial cancers refractory to standard chemotherapy.

\section{Conflict of Interests}

All authors of this paper reported no financial interests or potential conflict of interests.

\section{Acknowledgments}

The authors thank Emiri Nakazawa and Eri Saka for their skillful technical assistance and Mr. Kim Barrymore for English correction of this paper. This study was supported in part by a Grant-in-Aid for Clinical Cancer Research from the Ministry of Health, Labor and Welfare and a Grant-in-Aid from the Ministry of Education, Culture, Sports, Science and Technology of Japan (Grant no. 17390441 to T. Tsukamoto) and research grants from the Stiftelsen Japanese-Swedish Research Foundation.

\section{References}

[1] R. Siegel, D. Naishadham, and A. Jemal, "Cancer statistics, 2013," CA: A Cancer Journal for Clinicians, vol. 63, no. 1, pp. 11-30.

[2] National Comprehensive Cancer Network, NCCN Clinical Practice Guidelines in Oncology: Bladder Cancer V.1, 2013, http:// www.nccn.org/professionals/physician_gls/pdf/bladder.pdf.

[3] A. Stenzl, N. C. Cowan, M. De Santis et al., "Treatment of muscle-invasive and metastatic bladder cancer: update of the EAU guidelines," European Urology, vol. 59, no. 6, pp. 1009-1018, 2011.

[4] H. Kitamura, K. Taguchi, Y. Kunishima et al., "Paclitaxel, ifosfamide, and nedaplatin as second-line treatment for patients with metastatic urothelial carcinoma: a phase II study of the SUOC group," Cancer Science, vol. 102, no. 6, pp. 1171-1175, 2011.

[5] P. Albers, S.-I. Park, G. Niegisch et al., "Randomized phase III trial of 2nd line gemcitabine and paclitaxel chemotherapy in patients with advanced bladder cancer: Short-term versus prolonged treatment [German Association of Urological Oncology
(AUO) trial AB 20/99]," Annals of Oncology, vol. 22, no. 2, pp. 288-294, 2011.

[6] J. Bellmunt, C. Théodore, T. Demkov et al., "Phase III trial of vinflunine plus best supportive care compared with best supportive care alone after a platinum-containing regimen in patients with advanced transitional cell carcinoma of the urothelial tract," Journal of Clinical Oncology, vol. 27, no. 27, pp. 4454-4461, 2009.

[7] S. A. Rosenberg, "A new era for cancer immunotherapy based on the genes that encode cancer antigens," Immunity, vol. 10, no. 3, pp. 281-287, 1999.

[8] S. A. Rosenberg, "Progress in human tumour immunology and immunotherapy," Nature, vol. 411, no. 6835, pp. 380-384, 2001.

[9] S. A. Rosenberg, J. C. Yang, and N. P. Restifo, "Cancer immunotherapy: moving beyond current vaccines," Nature Medicine, vol. 10, no. 9, pp. 909-915, 2004.

[10] Y. Hirohashi, T. Torigoe, A. Maeda et al., "An HLA-A24-restricted cytotoxic T lymphocyte epitope of a tumor-associated protein, survivin," Clinical Cancer Research, vol. 8, no. 6, pp. 17311739, 2002.

[11] H. Kitamura, T. Torigoe, I. Honma et al., "Expression and antigenicity of survivin, an inhibitor of apoptosis family member, in bladder cancer: implications for specific immunotherapy," Urology, vol. 67, no. 5, pp. 955-959, 2006.

[12] I. Honma, H. Kitamura, T. Torigoe et al., "Phase I clinical study of anti-apoptosis protein survivin-derived peptide vaccination for patients with advanced or recurrent urothelial cancer," Cancer Immunology, Immunotherapy, vol. 58, no. 11, pp. 1801-1807, 2009.

[13] H. Kameshima, T. Tsuruma, T. Torigoe et al., "Immunogenic enhancement and clinical effect by type-I interferon of anti-apoptotic protein, survivin-derived peptide vaccine, in advanced colorectal cancer patients," Cancer Science, vol. 102, no. 6, pp. 1181-1187, 2011.

[14] H. Kameshima, T. Tsuruma, G. Kutomi et al., "Immunotherapeutic benefit of a-interferon (IFN $\alpha$ ) in survivin2B-derived peptide vaccination for advanced pancreatic cancer patients," Cancer Science, vol. 104, no. 1, pp. 124-129, 2013.

[15] National Cancer Institute, Cancer Therapy Evaluation Program, "Common Terminology Criteria for Adverse Events (CTCAE) Version 4.0," http://evs.nci.nih.gov/ftp1/CTCAE/CTCAE_4.03_ 2010-06-14_QuickReference_5x7.pdf.

[16] K. Palucka, H. Ueno, and J. Banchereau, "Recent developments in cancer vaccines," Journal of Immunology, vol. 186, no. 3, pp. 1325-1331, 2011.

[17] A. S. Bear, C. R. Cruz, and A. E. Foster, "T cells as vehicles for cancer vaccination," Journal of Biomedicine and Biotechnology, vol. 2011, Article ID 417403, 7 pages, 2011.

[18] L. Höltl, R. Ramoner, C. Zelle-Rieser et al., "Allogeneic dendritic cell vaccination against metastatic renal cell carcinoma with or without cyclophosphamide," Cancer Immunology, Immunotherapy, vol. 54, no. 7, pp. 663-670, 2005.

[19] J.-Y. Liu, Y. Wu, X.-S. Zhang et al., "Single administration of low dose cyclophosphamide augments the antitumor effect of dendritic cell vaccine," Cancer Immunology, Immunotherapy, vol. 56, no. 10, pp. 1597-1604, 2007.

[20] R. Okita, Y. Yamaguchi, M. Ohara et al., "Targeting of CD4+CD25high cells while preserving CD4+CD25low cells with low-dose chimeric anti-CD25 antibody in adoptive immunotherapy of cancer," International Journal of Oncology, vol. 34, no. 2, pp. 563-572, 2009. 
[21] T. Ghansah, N. Vohra, K. Kinney et al., "Dendritic cell immunotherapy combined with gemcitabine chemotherapy enhances survival in a murine model of pancreatic carcinoma," Cancer Immunology and Immunotherapy, vol. 62, no. 6, pp. 1083-1091, 2013.

[22] United States Food and Drug Administration, "Guidance for Industry: Clinical Considerations for Therapeutic Cancer Vaccines," http://www.fda.gov/downloads/BiologicsBloodVaccines/GuidanceComplianceRegulatoryInformation/Guidances/Vaccines/UCM278673.pdf. 


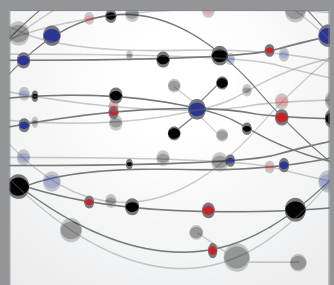

The Scientific World Journal
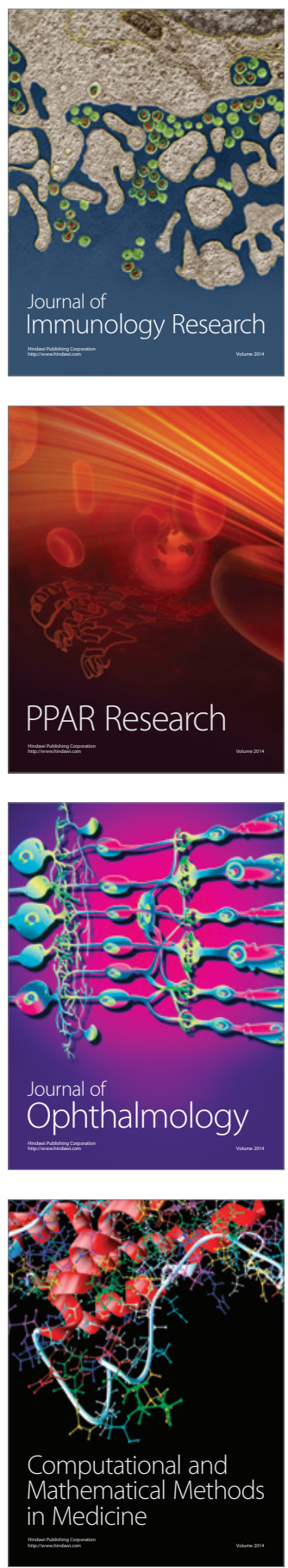

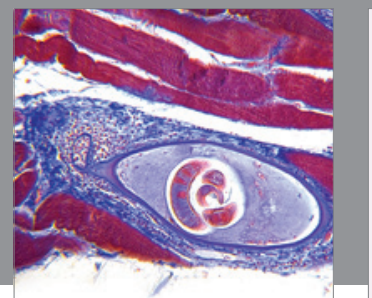

Gastroenterology

Research and Practice
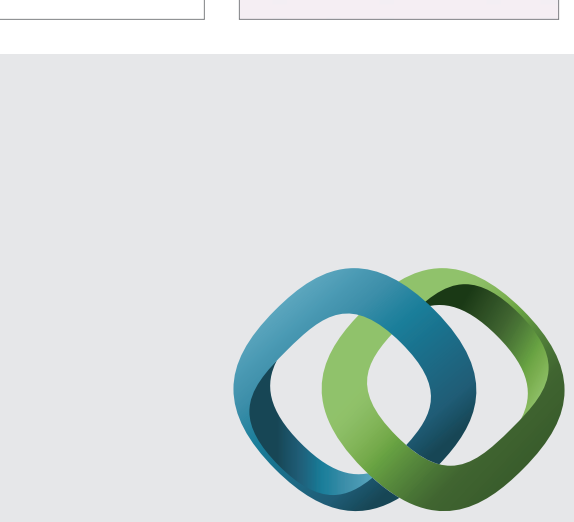

\section{Hindawi}

Submit your manuscripts at

http://www.hindawi.com
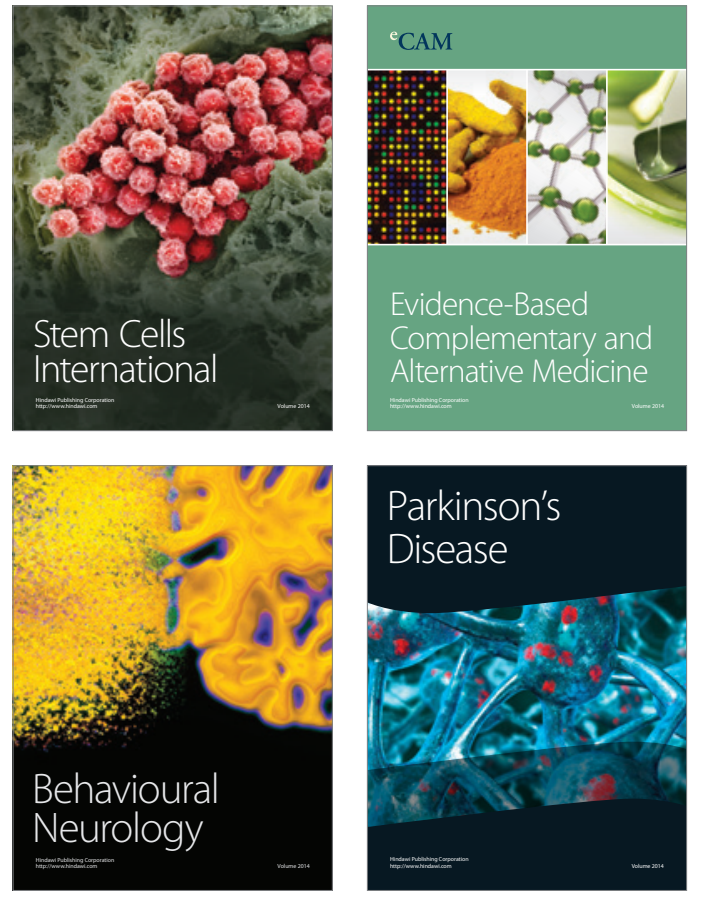
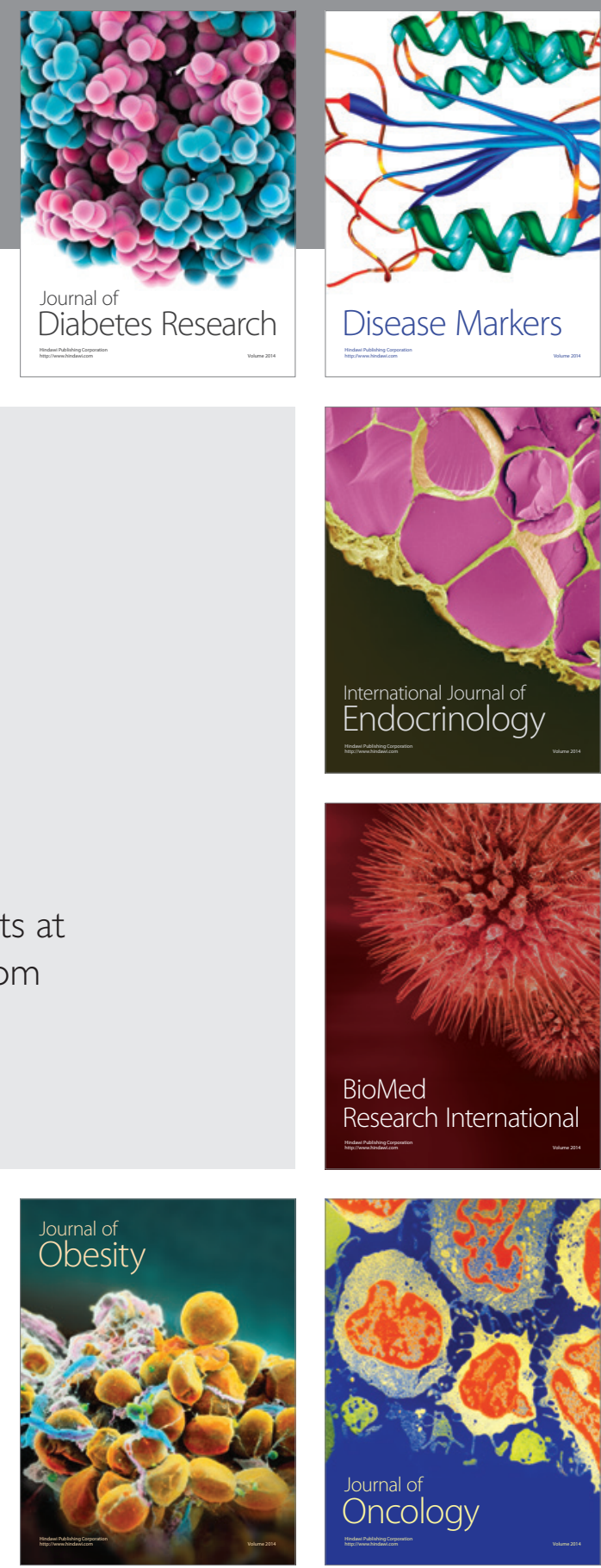

Disease Markers
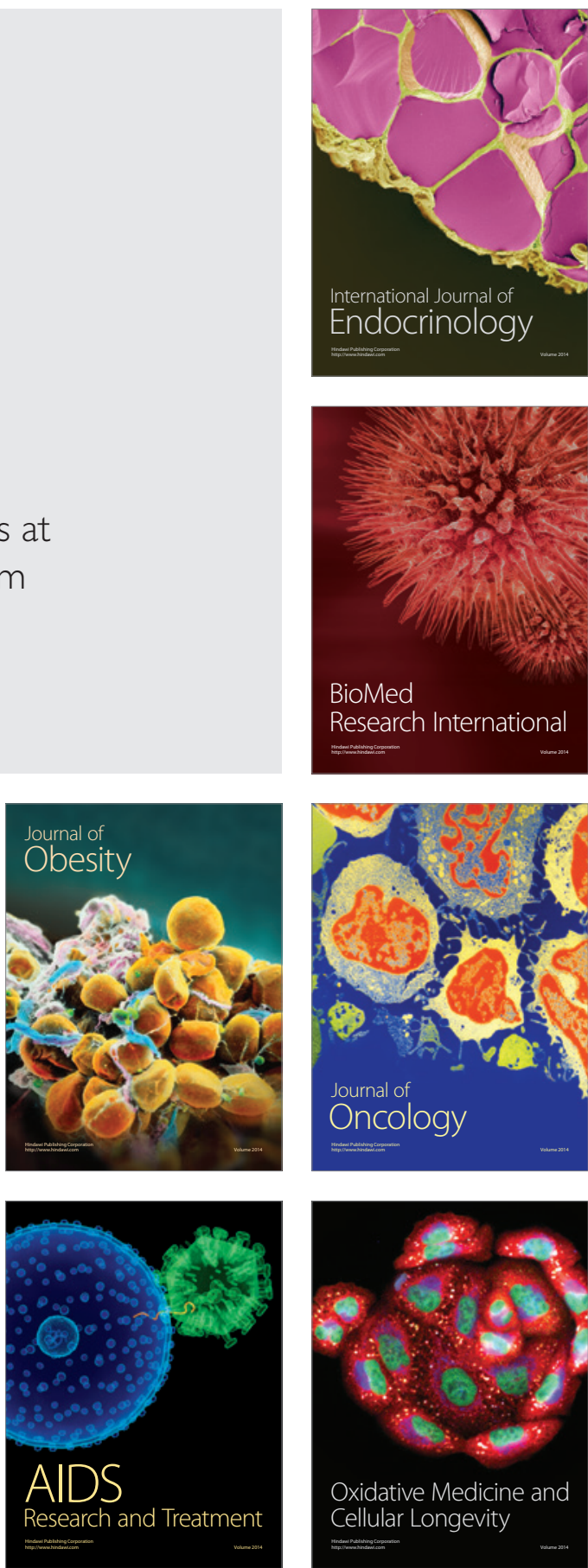